\title{
Avaliação da funcionalidade e força muscular periférica pós desmame da ventilação mecânica em uma UTI adulto
}

\author{
Evaluation of peripheral muscle strength and functionality after mechanical ventilation in an adult
} ICU

\author{
Evaluación de la fuerza y funcionalidad de los músculos periféricos después de la ventilación \\ mecánica en una UCI de adultos
}

\section{Resumo}

Este estudo visa avaliar a força muscular e a funcionalidade de pacientes pós desmame da ventilação mecânica (VM) em uma unidade de terapia intensiva adulto. Trata-se de uma pesquisa quantitativa e retrospectiva, através de prontuários de pacientes hospitalizados no período de junho de 2019 a março de 2021 de ambos os sexos, com idades entre 18 a 83 anos aprovada pelo Comitê de Ética em Pesquisa. Utilizou-se um questionário criado pelos próprios autores que inclui: desmame, tempo da VM, força muscular por dinanometria e funcionalidade dos pacientes. Analisada estatisticamente através do software BioEstat. 5.0. Foram utilizados 106 prontuários sobre o tipo de desmame, com predominância a prolongado, com permanência extendida de 8 a 14 dias em VM. Acerca da força muscular periférica 34,9\% da amostra obteve dinanometria de 6 a $10 \mathrm{Kg} / \mathrm{F}$ caracterizando fraqueza muscular. Quanto à funcionalidade, $34 \%$ dos pacientes saíram em sedestação beira leito e 27,4\% deambulando; porém 21,7\% continuaram acamados, expressando funcionalidade heterogênea que pode ser explicada pela alta prematura. Observou-se uma força muscular periférica reduzida, o que dificultou o desmame da VM predominando o desmame prolongado, o que possivelmente reflete redução da capacidade funcional dos pacientes.

Palavras-chave: Desmame; Ventilação mecânica; Fraqueza muscular; Estado funcional.

\begin{abstract}
The purpose of this study is to evaluate the muscle strength and patients' functionality after weaning from mechanical ventilation (MV) in an adult intensive care unit. This is a quantitative and retrospective study that analyzed the medical records of patients hospitalized from June 2019 to march 2021, both genders, aged 18 and 83 years old, approved by the Research Ethics Committee. A questionnaire created by the authors was used, which includes: weaninng, time on MV, muscle strenght by dynamometry, and patients' functionality. Statistical analysis using BioEstat 5.0 software.106 medical records were used on the type of weaning, with prolonged weaning, with a stay of 8 to 14 days on MV. Regarding peripheral muscle strength, 34,9\% of the sample had a dynamometry of 6 to $10 \mathrm{Kg} / \mathrm{F}$, characterizing muscle weakness. About functionality $34 \%$ of patients were in bedside sitting and $27.4 \%$ walked, but $21.7 \%$ remained bedridden, expressing heterogeneous functionality that could be expounded by premature discharge. The study has shown reduced peripheral muscle strength, which made it difficult to wean from MV, predominantly prolonged weaning, which possibly reflects a reduction in the patients' functional capacity.
\end{abstract}

Keywords: Weaning; Mechanical ventilation; Muscle weakness; Functional state. 


\begin{abstract}
Resumen
Este estudio tiene como objetivo evaluar la fuerza muscular y la funcionalidad de los pacientes después del destete de la ventilación mecánica (VM) en una unidad de cuidados intensivos para adultos. Se trata de una investigación cuantitativa y retrospectiva, utilizando historias clínicas de pacientes hospitalizados desde junio de 2019 a marzo de 2021 de ambos sexos, con edades comprendidas entre 18 y 83 años, aprobadas por el Comité de Ética en Investigación. Se utilizó un cuestionario elaborado por los autores que incluye: destete, tiempo en VM, fuerza muscular por dinanometría y funcionalidad del paciente. Analizado estadísticamente mediante el software BioEstat. 5,0. Se utilizaron 106 registros sobre el tipo de destete, predominantemente prolongado, con una estancia prolongada de 8 a 14 días en VM. En cuanto a la fuerza de los músculos periféricos, el 34,9\% de la muestra obtuvo una dinanometría de 6 a $10 \mathrm{~kg} /$ F, caracterizando la debilidad muscular. En cuanto a la funcionalidad, el 34\% de los pacientes se quedó sentado junto a la cama y el 27,4\% caminó; sin embargo, el 21,7\% permaneció postrado en cama, expresando una funcionalidad heterogénea que puede explicarse por el alta prematura. Se observó una reducción de la fuerza de los músculos periféricos, lo que dificultaba el destete de la VM, predominantemente un destete prolongado, lo que posiblemente refleja una reducción de la capacidad funcional de los pacientes.
\end{abstract}

Palabras clave: Destete, Ventilación mecánica, Debilidad muscular, Estado funcional.

\title{
1. Introdução
}

A unidade de terapia intensiva (UTI) é caracterizada como um ambiente designado aos cuidados da saúde de pacientes críticos, detendo novas tecnologias e atendimentos especializados, onde os profissionais e os dispositivos de assistência são essenciais para manter a vitalidade dos indivíduos (Eugênio; Filho \& Sousa, 2017). Na unidade crítica são encontrados pacientes submetidos a ventilação mecânica invasiva e sob efeito de sedativos para facilitar a intubação e atenuar a crise respiratória, bloqueadores neuromusculares que neutralizam atividade muscular e antibióticos que atacam bactérias que são prejudiciais ao organismo (Lima, 2017).

Os critérios de ocupação de leito na unidade de terapia intensiva são norteados em vários fatores, existindo a possibilidade de encontrar pacientes graves com vários sistemas acometidos, ou o oposto como sujeitos que estão confortáveis sem necessidade de suportes invasivos ou oxigênio suplementar. Dessa forma, o principal objetivo da unidade crítica é monitorização contínua do indivíduo, permitindo adiantar condutas em pacientes que possuam risco de vida (Moreira, 2020).

Da mesma maneira, na UTI é comum observamos pacientes recebendo suporte ventilatório invasivo, não invasivo, drogas vasoativas, antimicrobianos, sedação e analgesia em infusão contínua, e até mesmo procedimentos que auxiliam o retorno da funcionalidade, ou seja, esse local permite a ação de condutas extremas, direcionados por vezes em pacientes graves, ou indivíduos em estado de recuperação (Martins; Blais \& Leite, 2004). Já sobre o suporte pressórico em unidades críticas segundo Ouellette, et al. (2017) a ventilação mecânica invasiva é um método de suporte ventilatório, que busca sustentar o paciente até o momento que o mesmo esteja apto para ser devolvido para a respiração espontânea. Essa afirmativa reforça a utilização de protocolos de desmame, termo que defini o processo gradual de retirada da VM para a respiração natural.

Conhecendo o perfil da unidade de terapia intensiva que trabalha com pacientes extremamente graves como também indivíduos em recuperação, faz-se necessário contabilizar o tempo de estadia na unidade. Logo, o tempo de permanência é um indicador que reflete a eficácia da assistência aos pacientes, pois quanto maior o tempo de confinamento nas unidades piores serão os desfechos clínicos que por vezes está relacionado com a ineficiência dos cuidados assistenciais e o desmame prolongado (Martins; Blais \& Leite, 2004).

Além disso, o período prolongado na unidade de terapia intensiva coincide com um somatório de fatores negativos. Para Abelha, et al. (2006) a taxa de permanência dos pacientes críticos em unidade de terapia brasileira varia entre 4 a 10 dias, esse tempo contribui para o aumento de número de infecções, aumento dos custos hospitalares, fraqueza muscular adquirida na UTI, declínio funcional, delírio e mortalidade.

Entre as complicações citadas, devido ao período de internação, a principal delas é a fraqueza muscular adquirida na UTI, essa complicação contribui para fatores adversos na pós-alta como alterações psíquicas, distúrbios do sono e dificuldade 
para retornar as atividades laborais. A ocorrência de distúrbios neuromusculares e miopatias podem alcançar uma taxa de até $80 \%$ nos indivíduos gravemente enfermos. Essa taxa elevada de doentes fracos está intimamente ligada com o maior tempo de permanência na ventilação mecânica, além de subtrair aqueles que serão os sobreviventes de uma UTI (Vanhorebeek; Latronico $\&$ Berghe, 2020).

A perda de funcionalidade durante a internação também mostra íntima relação com o tempo de internação e o uso de suporte ventilatório invasivo, com o mesmo grau de relação que a fraqueza muscular adquirida na UTI. Consequentemente, o tempo elevado de estadia na unidade crítica acentua o prejuízo funcional, reduzindo a capacidade vital forçada, o pico de fluxo expiratório (tosse) e a capacidade de ultrapassar um teste respiração espontânea, favorecendo a ventilação prolongada (Martinez, Bispo, Duarte \& Neto, 2014). Este prejuízo por sua vez transpassa o período de internação levando consequências mesmo no período pós-alta, reforçando a incidência de síndrome pós-terapia intensiva e reinternações (Vesz et al., 2013).

Portanto, a doença crítica não finaliza com a alta da UTI. Ela persiste no âmbito extra-hospitalar amplificando as reinternações, assim como reduzindo a capacidade funcional para atividades de vida diária do paciente. Segundo Rawal, Yadal e Kumar (2017) a síndrome pós-terapia intensiva (SPTI), na língua inglesa chamada de Post-Intensive Care Syndrome (PICS) é definida como uma complicação da doença crítica que persiste além da alta hospitalar ocasionada pelo tempo elevado de permanência na unidade. Esta patologia se manifesta de várias maneiras que inclui desde a perda física até o prejuízo mental. A SPTI explica que indivíduos com longa internação poderão desenvolver além dos comprometimentos cognitivos, hipoglicemia, hiperglicemia, hipoxemia, hipotensão, dificuldade de mobilidade, prejuízo no ato tussígeno, insônia, desconforto respiratório entre outras complicações (Santos, Neves, Silva, Moura \& Coelho, 2020; Colbenson, Johnson \& Wilson, 2019).

Diante disso, ressaltamos o objetivo desse trabalho que busca investigar qual a força muscular periférica e a funcionalidade dos pacientes na alta da unidade hospitalar, além de avaliar as causas de permanência da UTI adulto após o desmame da ventilação mecânica.

\section{Metodologia}

Trata-se de uma pesquisa quantitativa, de caráter descritivo, de característica documental, possuindo uma cronologia transversal e retrospectiva. A população estudada é composta de indivíduos com idade entre 18 a 83 anos, de ambos os sexos, admitidos na UTI do Hospital do Baixo Amazonas. A amostra foi composta através de análise de documentos, por meio de prontuários do período de junho de 2019 a março de 2021. As informações obtidas através dos prontuários foram registradas em uma ficha criada pelos autores da pesquisa.

Os critérios de inclusão do estudo foram: pacientes adultos (18 - 83 anos); com dados de tempo de ventilação invasiva mínima de 24 horas, que conseguiram receber alta (melhorados ou curados) da unidade de terapia intensiva. Os instrumentos validados aplicados são a dinamometria de preensão palmar com valores de ponto de corte de $11 \mathrm{~kg} / \mathrm{F}$ para homens e $7 \mathrm{Kg} / \mathrm{F}$ para mulheres que configuram em fraqueza muscular ligada ao processo de internação. Em relação a escala de funcionalidade, o hospital não faz uso de nenhuma escala, contudo foi utilizado palavras-chaves da escala IMS na descrição dos prontuários.

Os dados adquiridos foram tabulados em planilha eletrônica por meio do software Microsoft Office Excel 2016. Os dados arquivados foram confrontados estatisticamente por meio da avaliação dos resultados através do software BioEstat 5.0, para verificar o nível de significância estatística, foi adotado nível de significância de 5\% (p<0,05) e nível de confiança correspondentes a $95 \%$.

O estudo seguiu a resolução $\mathrm{n}^{\circ}$ 466/12 CNS/CONEP que rege a pesquisa com seres humanos, CAAE: 45203221.9.0000.5168. Esta norma implica no anonimato dos indivíduos e no sigilo das informações adquiridas. Além disso, a pesquisa seguiu a Resolução de Julho de 2017, artigo $2^{\circ}$ onde explica o Termo de Compromisso de Uso de Dados (TCUD), 
afirmando que o estudo se compromete com o sigilo e a privacidade dos dados da pesquisa, assim como com o compromisso de que serão utilizados apenas para o estudo em questão.

\section{Resultados}

Foram avaliados 106 prontuários de pacientes submetidos a desmame da ventilação mecânica na UTI adulto do Hospital. Observou-se a prevalência do gênero masculino, com 56,6\% (n=60), e 43,4\% ( $n=46)$ foram do gênero feminino, demonstrando uma amostra não homogênea quanto ao gênero. Além disso, a média de idade dos pacientes extubados foi de 51,6 anos com desvio padrão de $\pm 17,2$ anos da amostra. (Tabela 1).

Tabela 1: Caracterização sociodemográficas da amostra, n=106. Santarém - Pará, 2021.

\begin{tabular}{cccc}
\hline Variável & N & $\mathbf{\%}$ & p-valor \\
\hline Gênero & & & \\
Feminino & 46 & $43,4 \%$ & 0,2067 \\
Masculino & 60 & $56,6 \%$ & \\
Faixa-etária & & & $<0,0001^{*}$ \\
Menor que 20 anos & 3 & $2,8 \%$ & \\
De 20 a 29 anos & 11 & $10,4 \%$ & \\
De 30 a 39 anos & 20 & $18,9 \%$ & \\
De 40 a 49 anos & 9 & $8,5 \%$ & \\
De 50 a 59 anos & 18 & $29,2 \%$ & \\
De 60 a 69 anos & 31 & $10,4 \%$ & \\
De 70 a 79 anos & 11 & $2,8 \%$ & \\
80 anos ou mais & 3 & & \\
& & $51,6 \pm 17,2$ & \\
Média aritmética \pm desvio-padrão (idade) & &
\end{tabular}

Fonte: Gomes et al. (2021).

Em relação às causas que levaram a internação, $50 \%(n=53)$ da amostra era composto por pacientes acometidos pela COVID-19, tendo em vista o período da coleta, com esse grupo ocupando a maior parte da amostragem (Tabela 2). Já em relação ao motivo que levou a necessidade de via aérea artificial (Tabela 3), 66\% $(n=70)$ foram por insuficiência respiratória tipo III, causa relacionada com o item anterior (perfil da amostra). Do mesmo modo, a amostra foi dividida em tipos de desmame, onde a maior amostragem compõe o desmame prolongado, 31,1\% ( $\mathrm{n}=33)$ (Tabela 3). Ressalta-se também, que em 31,3\% ( $\mathrm{n}=33)$, da amostragem permanecem na unidade crítica entre 8 a 14 dias. Já os dados referentes aos pacientes que não foram extubados e sim desmamados com a utilização de traqueostomia, compõe 19,8\% $(n=21)$ do total. Quando classificados com o tempo de saída da ventilação da mecânica após a confecção da traqueostomia, $80,2 \%(n=85)$, conseguiram sair da pressão positiva nas primeiras 24 horas após a colocação da prótese artificial. 
Tabela 2: Causas de internação na unidade de terapia intensiva, n=106. Santarém - Pará, 2021.

\begin{tabular}{|c|c|c|c|}
\hline Características clínicas da amostra & Amostra $(n=106)$ & $\%$ & p-valor \\
\hline \multicolumn{4}{|l|}{ Diagnóstico principal } \\
\hline Asma grave & 1 & $0,9 \%$ & $<0,0001 *$ \\
\hline Doenças de gravidez & 1 & $0,9 \%$ & \\
\hline Doença do aparelho digestivo & 3 & $2,8 \%$ & \\
\hline Choque séptico & 5 & $4,7 \%$ & \\
\hline Doença autoimune & 5 & $4,7 \%$ & \\
\hline Oncológico & 17 & $16,0 \%$ & \\
\hline Doença cardiovascular & 21 & $19,8 \%$ & \\
\hline Coronavírus & 53 & $50,0 \%$ & \\
\hline
\end{tabular}

Fonte: Autores.

Tabela 3: Necessidade e tempo de permanência em ventilação mecânica (VM); desmame e dias até a saída da VM por traqueostomia (TQT); tempo de permanência na Unidade de Terapia Intensiva (UTI) após desmame da VM, n=106. Santarém Pará, 2021.

\begin{tabular}{|c|c|c|c|}
\hline Características relacionadas a Ventilação Mecânica & Amostra $(n=106)$ & $\%$ & p-valor \\
\hline \multicolumn{4}{|l|}{ Motivo ou necessidade de VM } \\
\hline Convulsão & 1 & $0,9 \%$ & $<0,0001 *$ \\
\hline Choque séptico & 2 & $1,9 \%$ & \\
\hline Cirurgia & 26 & $24,5 \%$ & \\
\hline IRPA-I & 6 & $5,7 \%$ & \\
\hline IRPA-III & 70 & $66,0 \%$ & \\
\hline IRPA-II & 1 & $0,9 \%$ & \\
\hline \multicolumn{4}{|l|}{ Tempo em (dias) em VM } \\
\hline Até 01 dia & 21 & $19,8 \%$ & $0,0018 *$ \\
\hline De 02 a 07 dias & 27 & $25,5 \%$ & \\
\hline De 08 a 14 dias & 33 & $31,1 \%$ & \\
\hline De 15 a 30 dias & 17 & $16,0 \%$ & \\
\hline Acima de 30 dias & 8 & $7,5 \%$ & \\
\hline Média aritmética \pm desvio-padrão & \multicolumn{2}{|c|}{$12,1 \pm 14,9$} & \\
\hline \multicolumn{4}{|l|}{ Desmame realizado p/ TQT } \\
\hline Sim & 21 & $19,8 \%$ & $<0,0001 *$ \\
\hline Não & 85 & $80,2 \%$ & \\
\hline \multicolumn{4}{|l|}{ Dias até a saída da VM via TQT } \\
\hline Menos de 01 dia & 85 & $80,2 \%$ & $<0,0001 *$ \\
\hline De 01 a 07 dias & 5 & $4,7 \%$ & \\
\hline De 08 a 14 dias & 9 & $8,5 \%$ & \\
\hline De 02 a 07 dias & 6 & $5,7 \%$ & \\
\hline Acima de 30 dias & 1 & $0,9 \%$ & \\
\hline Média aritmética \pm desvio-padrão & \multicolumn{2}{|c|}{$3,2 \pm 11,8$} & \\
\hline \multicolumn{4}{|l|}{ Tempo em dias de permanência na UTI pós desmame da VM } \\
\hline Até 01 dia & 1 & $0,9 \%$ & $<0,0001 *$ \\
\hline De 02 a 07 dias & 64 & $60,4 \%$ & \\
\hline De 08 a 14 dias & 30 & $28,3 \%$ & \\
\hline De 15 a 30 dias & 9 & $8,5 \%$ & \\
\hline Acima de 30 dias & 2 & $1,9 \%$ & \\
\hline Média aritmética \pm desvio-padrão & \multicolumn{2}{|c|}{$8,0 \pm 6,0$} & \\
\hline
\end{tabular}

Fonte: Gomes et al. (2021).

A análise do desfecho da força muscular periférica no dia da alta unidade crítica evidenciou que 34,9\% (n=37) dos pacientes obtiveram força de preensão palmar através da dinamometria entre 6 a $10 \mathrm{~kg} / \mathrm{F}$, dado possivelmente relacionado com 
o perfil da amostra caracterizado por indivíduos de desmame difícil e prolongado, ou seja, aqueles sujeitos que na somatória ultrapassaram 7 dias de VM (Tabela 4). Já em relação ao maior grau de funcionalidade no dia da alta da UTI (Tabela 4), 34\% $(n=36)$, conseguiram controle de tronco total ou parcial com capacidade de sedestação beira-leito.

Tabela 4: Características sobre a força muscular e grau de funcionalidade após desmame da ventilação mecânica (VM), n=106, Santarém - Pará, 2021.

\begin{tabular}{|c|c|c|c|}
\hline & Amostra (n=106) & $\%$ & p-valor \\
\hline \multicolumn{4}{|l|}{ Informe dinamometria em quilograma-força $(\mathrm{Kg} / \mathrm{F})$} \\
\hline Acima de 40 & 6 & $5,7 \%$ & $<0,0001^{*}$ \\
\hline De 02 a 05 & 14 & $13,2 \%$ & \\
\hline De 06 a 10 & 37 & $34,9 \%$ & \\
\hline De 11 a 15 & 18 & $17,0 \%$ & \\
\hline De 16 a 20 & 14 & $13,2 \%$ & \\
\hline De 21 a 25 & 7 & $6,6 \%$ & \\
\hline De 26 a 30 & 6 & $5,7 \%$ & \\
\hline De 31 a 35 & 2 & $1,9 \%$ & \\
\hline De 36 a 40 & 2 & $1,9 \%$ & \\
\hline Média aritmética \pm desvio-padrão & 15,2 & 11,1 & \\
\hline \multicolumn{4}{|l|}{ Maior grau de Funcionalidade } \\
\hline $\begin{array}{l}\text { Acamado ou mudança de decúbito - Ativa, assistida e } \\
\text { passiva }\end{array}$ & 23 & $21,7 \%$ & $<0,0001^{*}$ \\
\hline Sedestação beira leito - Ativa ou assistida & 36 & $34,0 \%$ & \\
\hline Sedestação fora do leito & 8 & $7,5 \%$ & \\
\hline Ortostase & 10 & $9,4 \%$ & \\
\hline Deambular & 29 & $27,4 \%$ & \\
\hline
\end{tabular}

Fonte: Gomes et al. (2021).

Ressalta-se que quando correlacionado a idade da amostra com o tempo exposto a ventilação mecânica não foi evidenciado coeficiente positivo de relação $(\mathrm{r}=-0,0334)$, indicando que tantos indivíduos jovens como indivíduos mais idosos necessitaram de pressão de positiva por via invasiva. Tal achado se deve possivelmente pelo perfil da amostra, onde 50\% ( $\mathrm{n}=53)$ são compostas por pacientes infectados pela COVID-19 (Figura 1). Já quando confrontado a idade dos pacientes e o tempo de permanência na unidade crítica pós desmame da ventilação mecânica, também não foi evidenciado correlação positiva ( $\mathrm{r}=$ $0,1454)$, achado que poderia ser justificado pelo perfil de desmame da amostra, desmame difícil ou prolongado $(54 \%, \mathrm{n}=58)$ onde a instalação da doença crítica afeta tanto jovens como idosos.

Da mesma maneira quando estabelecido inter-relação entre a idade da amostra e a força muscular periférica no dia da alta, também não se observou coeficiente positivo $(r=-0,0392)$, dados que poderiam ser justificados da mesma maneira que no item anterior (Figura 1). 
Figura 1: Resultados da relação da idade com o tempo em ventilação mecânica, saída da ventilação mecânica (VM) via traqueostomia (TQT), permanência na UTI pós desmame e força muscular, Santarém - Pará.



c) idade (anos) e Tempo em dias de permanência na UTI pós desmame da VM

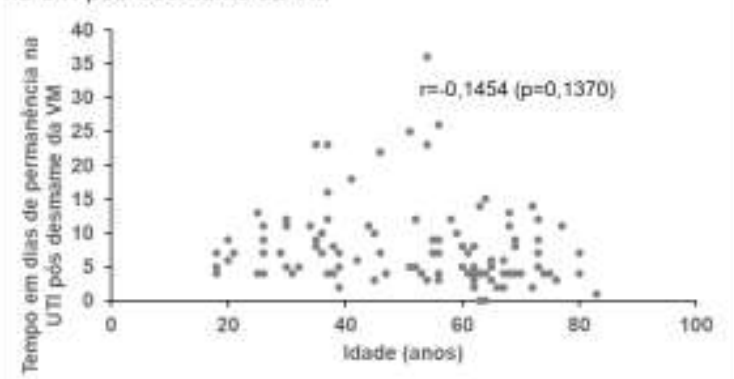

d) idade (anos) e dinanometria (Kg/F)

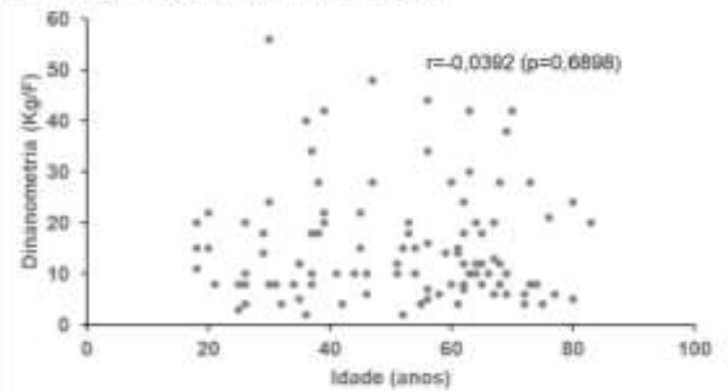

Fonte: Gomes et al. (2021).

Foi tentando estabelecer relação entre o tempo transcorrido em ventilação mecânica e a força muscular periférica no dia da alta, os achados do estudo mostraram uma relação também negativa ( $\mathrm{r}=-0,3458)$, por se tratar de uma amostra composta por pacientes em desmame difícil e prolongado $(54 \%, \mathrm{n}=58)$, os resultados se distanciam dos achados na literatura (Figura 2$)$. 
Figura 2: Resultados da relação do tempo em ventilação mecânica (VM) e saída da VM via traqueostomia (TQT) e permanência na UTI pós desmame e força muscular, Santarém - Pará, 2021.
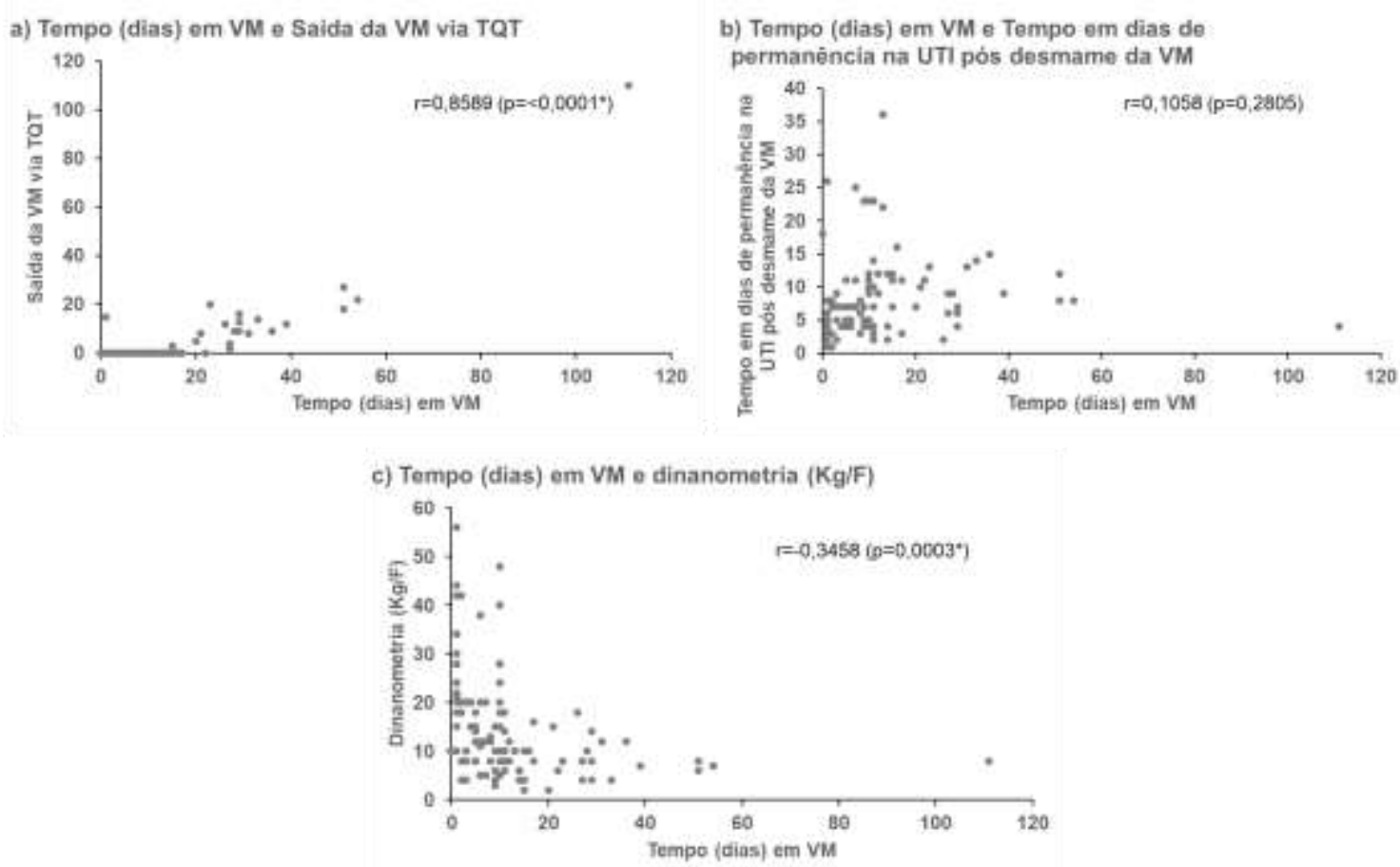

Fonte: Gomes et al. (2021).

Por fim, quando investigado conexão entre o tempo de permanência na UTI pós desmame da ventilação mecânica e a força muscular periférica no dia da alta, igualmente, não se evidenciou inter-relação positiva ( $\mathrm{r}=-0,1811)$, achado que poderia ser influenciado pelos protocolos de reabilitação da unidade crítica (Figura 3). 
Figura 3: Resultados da relação da saída da ventilação mecânica (VM) via traqueostomia (TQT) e permanência na UTI pós desmame e força muscular, Santarém - Pará, 2021.


Fonte: Gomes et al. (2021).

\section{Discussão}

No Brasil, a média de idade dos pacientes internados em unidade de terapia intensiva adulto está entre 40 e 69 anos, com predominância do sexo masculino, dados que corroboram com os achados encontrados no presente estudo (Rodriguez, et al., 2015). Contudo, dados recentes relacionados com a pandemia da COVID-19, a média de idade aumentou para acima de 65 anos, com a mesma predominância para o sexo masculino, dados próximos a média encontrada no estudo em relação à média nacional (Souza, Hojo, Batista, Silva \& Guidoni, 2021).

Quando classificadas as UTIs pela necessidade de internação, mais de 50\% dos motivos de estadia são por desordens ou acometimento cardiorrespiratório (Feijó Car et al., 2006). Contribuindo com os dados da comunidade científica, nosso estudo encontrou que 50\% dos pacientes internados eram por desordens respiratórias, contudo, a amostra foi analisada durante a pandemia do COVID-19, justificando então os motivos de internação por esta causa. Da mesma forma, quando investigado dados de internação nas unidades críticas nos anos de 2020 e 2021 foram encontrados elementos que justificam a causa de internação primária como insuficiência respiratória tipo III, comprovada pela sobrecarga de internação de pacientes COVID-19, estes dados respaldam com os dados encontrados, pois $66 \%$ da causa de hospitalização foi insuficiência respiratória mista (Souza et al., 2021).

Segundo Esteban, et al. (1999) em relação a saída da ventilação mecânica invasiva 40\% do tempo dos pacientes em VM é gasto durante o desmame, contudo, uma parcela inferior a um terço passará por um desmame prolongado, estes possuem alta taxa de mortalidade (Boles et al., 2007). Os dados investigados encontraram mais de 50\% (54\%, $\mathrm{n}=58)$ dos pacientes classificados como desmame difícil e prolongado, tal achado é justificado, pois a metade da amostra foi composta com pacientes de síndrome do desconforto respiratório agudo (SDRA) moderado e grave, que evoluíram com necessidade de sedação profunda e uso de bloqueadores neuromusculares, impossibilitando a extubação no primeiro teste de respiração espontânea, ou seja, de 
forma precoce. Estes resultados são semelhantes aos encontrados na literatura, onde indivíduos com SDRA na categorização moderado e grave evoluíram em sua maioria para traqueostomia e desmame gradual da ventilação mecânica (Bordon et al., 2021). Semelhantemente, quando investigado o desmame da ventilação mecânica em indivíduos com SDRA devido infecção do COVID-19 encontrou-se que mais de 40\% dos pacientes necessitaram de mais um teste de respiração para alcançar a extubação, ou atingiram a saída da ventilação mecânica após 7 dias (Bordon et al., 2021; Nash et al., 2020).

Analisando ainda o tema desmame difícil e prolongado, boa parte dos pacientes evoluirá para traqueostomia, principalmente na presença de síndrome do desconforto respiratório agudo, idade avançada e comorbidades (Jaeger; Littlewood \& Durbin, 2002). Vários estudos preveem achados para colocação da traqueostomia para facilitar o desmame, entre eles estão: presença de injúria cerebral grave com incapacidade de proteção de vias aéreas, obstrução de vias aéreas superiores e falhas nos testes de respiração espontânea com necessidade de desmame gradual (Abe et al., 2018). Em contrapartida, a análise mostrou que apenas 19,8\% foram para traqueostomia e em um tempo máximo de 24 horas conseguiram desmame da pressão positiva (80,2\%), resultados que se opõem aos dados para pacientes em desmame difícil e prolongado (Boles et al., 2007).

Percebe-se, então, que parte dos pacientes que se encontravam em desmame prolongado e difícil não caminhou para traqueostomia e uma parcela foram extubados. Tal fato, pode ser justificado que durante a pandemia da COVID-19 a traqueostomia foi retardada devido ao risco de contaminação dos profissionais por partículas suspensas, sendo orientada a realização em até 21 dias, isso possibilitou a utilização de teste de respiração espontânea após 15 dias de intubação e por vezes gerando uma saída com sucesso da ventilação mecânica (Kwak, Connors \& Benedict, 2021). Outra possibilidade seria a perda da proteção de vias aéreas, onde é possível encontrar sucesso de desmame e insucesso de extubação, e quando colocado esses pacientes em traqueostomia, a saída da ventilação mecânica é realizada de forma quase imediata (Abe et al., 2018).

A alta da unidade crítica não significa que não ocorrerão novas internações, para isso preditores podem e devem ser usados para definir indivíduos com maior risco. Evidências demonstram que os indivíduos desmamados da ventilação mecânica invasiva que alcançam $23 \mathrm{Kg} / \mathrm{F}$ na avaliação da força muscular periférica com dinamômetro de preensão palmar estão com ganho para controlar o tronco, proteger suas vias aéreas e, na maioria dos casos, atingir a deambulação (Santos L.J. et al., 2017). Já nessa análise foi observada que a maioria dos pacientes se encontrava com uma força de preensão palmar inferior a $11 \mathrm{~kg} / \mathrm{F}$ (34\%), indicando fraqueza muscular adquirida na UTI. Essa relação justifica também o maior grau de funcionalidade encontrado, o controle de tronco com sedestação beira-leito ( $34 \%$ da amostra analisada).

Em afirmação com a citação anterior, quando investigado a força de preensão palmar em pacientes com desmame prolongado, os resultados encontrados também são inferiores a $11 \mathrm{~kg} / \mathrm{F}$, fato reforçado na literatura pela exposição prolongada de sedativos e uso de corticosteroides (Chlan, 2015). Embora a perda de massa muscular não seja um marcador sensível e específico para indicar mortalidade, quando examinado indivíduos internados concomitantemente com COVID-19 e SDRA, é salientado que a redução de massa muscular é um preditor de internação em UTI (Giraudo et al., 2021). Quando estabelecido inter-relação entre a idade da amostra e a força muscular periférica no dia da alta não foi observado correlação positiva, haja vista que, uma grande parcela da amostra analisada se encontrava em desmame difícil e prolongado, e apenas isso já é suficiente para reduzir a força de preensão palmar, independentemente da idade do indivíduo (Chlan, 2015).

Quando estabelecido relação entre o tempo de permanência em ventilação mecânica e a força muscular periférica, os achados do estudo mostraram uma relação também negativa ( $r=$ - 03458), isso entra em contraponto com a literatura já mencionada, pois quanto maior o tempo de ventilação, os estudos demonstram menor força periférica encontrada (Godoy et al., 2015). Contudo, parte da amostra analisada permaneceu na unidade por um tempo acima de 7 dias mesmo após o desmame da ventilação mecânica, se os mesmos receberam protocolos para incrementar a força periférica justificaria os desfechos.

Com relação a capacidade funcional no dia da alta da UTI, evidenciou uma heterogeneidade sobre os achados onde apenas $27,4 \%$ saíram deambulando em contrapartida $21,7 \%$ acamados. Tais resultados são reafirmados devido ao impacto do 
surto de COVID-19 e a sobrecarga nos hospitais onde no início da pandemia o principal desfecho era a alta e a rotatividade dos leitos, e com o transcorrer foi substituída pelo grau de funcionalidade no dia da saída e o risco do paciente para uma nova internação (Giraudo et al., 2021). Da mesma forma, outro fator que pode ter influenciado a funcionalidade é a característica da amostra, onde a sua maioria possui o diagnóstico de SDRA e COVID-19, que possivelmente resultou na aplicação de mais sedação profunda e mobilidade mais restrita do que em outras patologias sucedendo implicações na recuperação (Prescott \& Girard, 2020).

Consequentemente ao tempo de internação, quando investigado o comprometimento da funcionalidade na fase pós-alta em indivíduos com desmame difícil e prolongado é encontrado na fase pós-hospitalar declínio da independência funcional, e ainda dificuldade para o retorno as atividades laborais mesmo após 6 meses transcorridos (Brown et al., 2019). Se confrontado os dados dessa análise é observado que a maioria dos pacientes se encontrava com uma força de preensão palmar inferior a 11 $\mathrm{kg} / \mathrm{F}(34 \%)$, indicando fraqueza muscular adquirida na UTI com alto risco para novas internações e dependência de um cuidador nos próximos meses, com alto risco para desenvolvimento de SPTI.

Semelhante quando investigado a força de preensão palmar em indivíduos com SDRA devido ao COVID-19, evidências reportam que mesmo após transcorrer 1 ano da alta da unidade crítica, os sobreviventes se mantinham fracos, com força periférica média de $70 \%$ do valor previsto. Além de que, a funcionalidade avaliada pelo índice de Barthel que pontua o escore sobre a independência de atividade de vida diária foi apenas alcançada na maioria dos sobreviventes após 3 meses da saída hospitalar (Robinson et al., 2018). A presente análise não avaliou escores pós-alta, contudo a força e a funcionalidade encontrada se assemelham com o estudo anterior, onde a força muscular periférica está inferior a $11 \mathrm{Kg} / \mathrm{F}$ e o paciente é incapaz de deambular no dia da alta. Esses dados reforçam o risco para desenvolvimento de síndrome pós-terapia intensiva nessa amostra.

Sobre o mesmo tema, as evidências de síndrome pós-terapia intensiva em indivíduos que evoluíram de forma concomitante com SDRA e COVID-19 são altas. Desfechos apresentados sugerem um valor de $86 \%$ para pacientes que não retornarão ao trabalho nos primeiros 3 meses, semelhantemente essa taxa continuará elevada mesmo após 1 ano. A justificativa para esses indivíduos não retornarem ao trabalho nos primeiros 90 dias é a reduzida condição física e funcional no dia da alta (Latronico et al., 2021). Esses dados sugerem que a amostra analisada também possuirá tal risco, haja vista, que o maior grau de funcionalidade encontrado foi o controle de tronco com sedestação beira-leito ( $34 \%$ da amostra analisada), esse grau funcional impossibilidade a deambulação, desfavorecendo a maioria das atividades laborais.

Em contrapartida, aos pacientes com baixa funcionalidade, a análise observou que 27,4\% dos pacientes alcançam no dia da alta a deambulação, isso pode ser justificado pela adesão de protocolos de mobilização precoce encontrados nas unidades críticas. Dentre os diversos fatores que podem mudar o desfecho funcional no dia da alta as terapêuticas que favorecem a retirada do paciente do leito, que incluem sedestação na poltrona, ortostase e deambulação, são as mais eficazes (Winkelman et al., 2018).

Por fim, o estudo possui suas limitações principalmente relacionadas com o tamanho da amostra, e ainda por se tratar de um estudo observacional. Contudo, fomenta a busca para o devido tema em pesquisas futuras.

\section{Conclusão}

A força muscular periférica reduzida encontrada na pesquisa reflete no tempo de VM de 8 a 14 dias dos pacientes, prejudicando a retirada do paciente da ventilação mecânica invasiva e favorecendo o perfil do desmame prolongado. Em relação à capacidade funcional observou-se que mais de um terço dos pacientes saiu em sedestação beira leito, porém a quinta parte continuou acamados, esses resultados mostram que a funcionalidade foi heterogênea provavelmente relacionada ao perfil da amostra, e os protocolos de mobilização utilizados na unidade crítica. Além disso, o grau máximo de funcionalidade pode ser 
influenciado por vários fatores, incluindo o tempo de permanência na unidade pós desmame da ventilação mecânica. Isto posto, sugere-se novos estudos para refutar os dados apresentados e para uma maior fonte de dados em relação ao tema proposto.

\section{Referências}

Abelha, J. F., Castro, M. A., Landeiro, N. M., Neves, A. M. \& Santos, C. C. (2006). Mortalidade e o tempo de internação em uma unidade de terapia intensiva cirúrgica. Rev. Bras. Anestesiol., 56(1):34-45.

Abe, T., Madotto, F., Pham, T., Nagata I., Uchida, M., Tamiya, N., Kurahashi, K., Bellani, G., \& Laffey, J. G. (2018). Epidemiology and patterns of tracheostomy practice in patients with acute respiratory distress syndrome in ICUs across 50 countries. Crit. Care Med, 22(195):13054-13070.

Boles, J. M., Bion, J., Connors, A., Herridge, M., Marsh, B., Melot, C, Pearl, R, Silverman, H, Stanchina, M, Vieillard-Baron, A., \& Welte, T (2007). Weaning from mechanical ventilation. Eur. Respir. J., 29(5): 1033-1056.

Bordon, J., Akca, O., Furmanek, S., Cavallari, R. S., Suliman, S., Aboelnasr, A, Sinanova, B., \& Ramirez, J.A. (2021). Acute Respiratory Distress Syndrome and Time to Weaning Off the Invasive Mechanical Ventilator among Patients with COVID-19 Pneumonia. J. Clin. Med., 10(2935):1-11.

Brown, S. M., Bose, S., Banner-Goodspeed, V., Beesley, S. J., Dinglas, V. D., Hopkins, R. O., Jackson, J. C., Mir-Kasimov, M., Needham, D. M. \& Sevin, C. M. (2019). Approaches to addressing post-intensive care syndrome among intensive care unit survivors. AnnalsATS, 16(8):947-956.

Chlan, L. L. (2015). Description of Peripheral Muscle Strength Measurement and Correlates of Muscle Weakness in Patients Receiving Prolonged Mechanical Ventilatory Support. Am J Crit Care, 24(6):91-98.

Colbenson, G. A., Johnson, A. \& Wilson, M. E. (2019). Post-intensive care syndrome: impact, prevention, and management. Breathe, 2019 , 15(2): 98-101.

Eugenio, C. S., Filho, M. C. B., \& Souza, E. N. (2017). Visita aberta em UTI adulto. Rev. Enferm. da UFSM, 7(3):539-549.

Esteban A., Alía I, Tobin M.J., Gil A., Gordo F., Valverdú I., Blanch L., Bonet A., Vásquez A., De Pablo R., Torres A., De La Cal M.A. \& Macías S. (1999). Effect of spontaneous breathing trial duration on outcome of attempts to discontinue mechanical ventilation. Spanish Lung Failure Collaborative Group. Am $J$ Respir Crit Care Med, 159(2): 512-8.

Feijó C. A. R., Leite F. O. J., Martins A. C. S., Furtado A. H. J., Cruz L. L. S. \& Meneses F. A. (2006). Gravidade dos Pacientes Admitidos à Unidade de Terapia Intensiva de um Hospital Universitário Brasileiro. Rev. Bras. Ter. Intensiva, 18(1): 18-20.

Godoy M. D. P., Costa H. L. L. S., Neto A. E. S., Serejo A. L. C., Souza L. C., Kalil M. R., Mota R. F., Monteiro L. F., Souza O. G., Freitas M. R. G. Bastos V. H., Novellino P. N., Matta A. P \& Orsini M. (2015). Fraqueza muscular adquirida na UTI (ICU-AW): efeitos sistêmicos da eletroestimulação neuromuscular. Rev. Bras. de Neurol., 51(4):110-112.

Giraudo C., Librizzi G., Fichera G., Motta R., Balestro E., Calabrese F., Caretta G., Cattelan A. M., Navalesi P., Pelloso M., Plebani M., Rea F., Vettor R., Vianello A. \& Stramare R. (2021). Reduced muscle mass as predictor of intensive care unit hospitalization in COVID-19 patients. Plos one,16(6):1-10.

Jaeger J. M., Littlewood K. A. \& Durbin C. G. JR. (2002). The role of tracheostomy in weaning from mechanical ventilation. Rev. Respir Care, 47(4):469-80.

Kwak P. E., Connors J. R., \& Benedict P. A. (2021). Early Outcomes From Early Tracheostomy for Patients With COVID-19. JAMA Otolaryngol Head Neck Surg, 147(3): 239-244.

Latronico N., Peli E., Calza S., Rodella F., Novelli M. P., Cella A., Marshall J., Needham D.M., Rasulo, F.A. \& Piva S. (2021). Physical, cognitive and mental health outcomes in 1-year survivors of COVID-19-associated ARDS. Thorax, 0:1-4.

Lima, A.M.S. (2017). Conhecimento dos fisioterapeutas atuantes em UTI adulto sobre as contraindicações à mobilização em pacientes críticos. Dissertação (Mestrado em Fisioterapia) - Universidade Federal de Pernambuco, Recife, 1-70.

Martinez, B. P, Bispo, A. O., Duarte, A. C. M., \& Neto, M. G. (2014). Declínio funcional em uma unidade de terapia intensiva (UTI). Rev. Insp., 5(1): 1-5.

Martins M., Blais R., \& Leite I. C. (2004). Mortalidade hospitalar e tempo de permanência: comparação entre hospitais públicos e privados na região de Ribeirão preto, São Paulo, Brasil. Cad. Saúde Pública. Rio de Janeiro, 20(2): 268-282.

Moreira R. S. (2020). Covid-19: unidades de terapia intensiva, ventiladores mecânicos e perfis latentes de mortalidade associados à letalidade no Brasil. Cad. Saúde Pública, 36(5):1-12.

Nash D. S., Khoundabi B., Azar G. M., Maleakmohammad, M., Jamaati, H., \& Hashemian, S. M. (2020). Beneficial Outcomes of Early Tracheostomy in Patients Requiring Prolonged Mechanical Ventilation. Tanaffos, 19(4): 350-355.

Ouellette D. R., Patel S., Girard T. D., Morris P. E., Schimidt G. A., Truwit J. D., Alhazzani W., Burns S. M., Epstein S. K., Esteban A., Fan E., Ferrer M., Fraser G. L., Gong M. N., Hough C. L., Mehta S., Nanchal R., Pawlik A. J., Schweickert W.D., Sessler C. N., Strom T. \& Kress J. P. (2017). Liberation From Mechanical Ventilation in Critically Ill Adults: An Official American College of Chest Physicians/American Thoracic Society Clinical Practice Guideline. Evidence-Based Medicine, 151(1): 166-180.

Prescott H. C. \& Girard T. D. (2020). Recovery From Severe COVID-19. JAMA, 324(8):739-740.

Rawal, G, Yadav, S., \& Kumar, R. (2017). Post-intensive care syndrome. Journal of Translational Internal Medicine, 5(2): 90-92. 
Research, Society and Development, v. 10, n. 13, e554101321477, 2021

(CC BY 4.0) | ISSN 2525-3409 | DOI: http://dx.doi.org/10.33448/rsd-v10i13.21477

Robinson C. C., Rosa R. G., Kochhann R., Schneider D., Sganzerla D., Dietrich C., Sanchez E. C., Dutra F. H., Oliveira M. Q., Anzolin L. B., Menezes S. F., Jeffman R., Souza D., Silva S. F., Cruz L. N., Boldo R., Cardoso J. R., Birriel D. C., Gamboa M. N., Machado A. S., Andrade J. M .S. , Alencar C., Teixeira M. C., Vieira S. R. R., Moreira F. C., Amaral A., Silveira A. P. M., Teles J. M. M., Oliveira D. C., Oliveira L. C. J., Castro L. C., Silva M. S., Neves R. T., Gomes R. A., Ribeiro C. M., Cavalcanti A. B., Oliveira R. P., Maccari J. G., Berto P. P, Martins L. A., Santos R. L. S., Ue L. Y., Hammes L. S., Sharshar T., Bozza F., Falavigna M. \& Teixeira C. (2018). Qualidade de vida pós-unidades de terapia intensiva: protocolo de estudo de corte multicêntrico para avaliação de desfechos em longo prazo em sobreviventes de internação em unidades de terapia intensiva brasileiras. Rev Bras Ter Intensiva, 30(4):405-413.

Rodrigues A. H, Bub M. B. C., Perão O. F., Zandonadi G. R. \& Hernández M. J. (2016) Características epidemiológicas e causas de óbitos em pacientes internados em terapia intensive. Rev Bras Enferm, 69(2): 229-34.

Santos G. L. S, Neves L. T. S., Silva L. B., Moura T. G. S. \& Coelho M. X. (2020). Abordagem fisioterapêutica na síndrome pós-terapia intensiva (SPTI): uma revisão de literatura. UNIVALE, 2(1): 2-11.

Santos L. J., Silveira F. S., Muller F. F., Araújo H. D., Comerlato J. B., Silva M. C. \& Silva P. B. (2017). Functional assessment of inpatients in the Intensive Care Unit of the University Hospital of Canoas. Rev. Fisioter Pesqui., 24(4): 437-443.

Souza F. S. H., Hojo N. S. S., Batista B. D. O., Silva C. M. \& Guidoni D. L. (2021). On the analysis of mortality risk factors for hospitalized COVID-19 patients: A data-driven study using the major Brazilian database. Plos One,16(3): 1-11.

Vanhorebeek I., Latronico N., \& Berghe G. V. D. (2020) ICU-acquired weakness. Intensive Care Med, 46(8):1-17.

Vesz P. S., Costanzi M., Stolnik D., Dietrich C., Freitas K. L. C., Silva L. A., Almeida C. S., Souza C. O., Ondere J., Souza D. L. S., Neves T. E. O., Meister M.V., Barbosa E. S., Paiva M. P., Carvalho T. S., Savi A., Maccari J. G., Cremonese R. V., Ribeiro M. C. \& Teixeira C. (2013). Aspectos funcionais e psicológicos imediatamente após alta da unidade de terapia intensiva: coorte prospectiva. Rev Bras Ter Intensiva, 25(3): 218-224.

Zorowitz R. D. (2016). ICU-acquired weakness: a rehabilitation perspective of diagnosis, treatment, and functional management. Chest, 150(4):966-71.

Winkelman C., Sattar A., Momotaz H., Johnson K. D., Morris P., Rowbotottom J. R., Thornton J. D., Feeney S., \& Levine A. (2018). Dose of early therapeutic mobility:does frequency or intensity matter? Biol Res Nurs, 20(5):522-530. 